\title{
Axillary Web Syndrome in Breast Cancer: A Prevalent But Under-Recognized Postoperative Complication
}

\author{
Susan R. Harris \\ Department of Physical Therapy, Faculty of Medicine, University of British Columbia, Vancouver, BC, Canada
}

Keywords

Axillary web syndrome . Breast cancer .

Lymphatic cording

\section{Summary}

The purpose of this review is to define axillary web syndrome (AWS) and describe its diagnosis and management. The following databases were searched through July 2017: PubMed, EMBASE (OvidSP), Cumulative Index for Nursing and Allied Health Literature, Physiotherapy Evidence Database, and Cochrane Database of Systematic Reviews. Search terms included 'axillary web syndrome', 'axillary cording', and 'lymphatic cording'. 49 articles were identified; 8 did not relate to breast cancer, and 3 were not in English. Of the remaining articles, the majority were case reports, case series, or descriptive reviews. 2 systematic reviews were located as well as 1 randomized trial, 6 prospective cohort studies, and 2 retrospective cohort studies. Although a common sequela after axillary surgery for breast cancer staging, AWS has been poorly described in the medical literature as to the underlying pathophysiology, diagnosis, and management. Interventions range from patient education and simple reassurance that the syndrome will resolve spontaneously to active physical or manual therapies to maintain upper extremity range-of-motion, especially adequate motion for undergoing radiation therapy. Oncologists, breast surgeons, family physicians, and oncology nurse practitioners that work with patients with breast cancer should educate them about this prevalent complication and inform them preoperatively about what to anticipate.

(c) 2018 S. Karger GmbH, Freiburg

\section{Introduction}

Breast cancer is the most common cancer diagnosis among women in developed and underdeveloped countries around the world [1]. With a trend toward follow-up care for patients with breast cancer increasingly being provided by family physicians [2, 3 ], primary care practitioners as well as oncologists and breast surgeons need to be aware of the potential sequelae of breast cancer treatments (e.g., upper extremity lymphedema, shoulder range-ofmotion restrictions, arm weakness).

One outcome of breast cancer treatment that is even more common than lymphedema but has been far less studied or described in the peer-reviewed literature, is the axillary web syndrome (AWS). First described in 2001 [4, 5], AWS - known also as axillary or lymphatic cording $[6,7]$ - is a fairly common outcome of axillary lymph node removal as part of the staging for breast cancer, although prevalence figures vary widely among studies that have reported them [4, 6-13]. Years after it was first reported, AWS continues to be labeled as a 'poorly understood syndrome' [12] with the quality of the evidence describing it based largely on case reports as well as on retrospective and prospective cohort studies.

To inform this review, the following databases were searched from their inception through July 2017: PubMed, EMBASE (OvidSP), CINAHL (Cumulative Index for Nursing and Allied Health Literature), PEDro (Physiotherapy Evidence Database), and the Cochrane Database of Systematic Reviews. Search terms included 'axillary web syndrome', 'axillary cording', and 'lymphatic cording'. A total of 49 articles were identified, 8 of which did not relate to breast cancer and 3 of which were not written in English. Of the remaining 38 articles, the majority were case reports, case series, or descriptive review articles. 2 systematic reviews on AWS were located as well as 1 randomized controlled trial, 6 prospective cohort studies, and 2 retrospective cohort studies.

\section{KARGER}

() 2018 S. Karger GmbH, Freiburg
Susan R. Harris, PhD, PT, FCAHS, Professor Emerita 


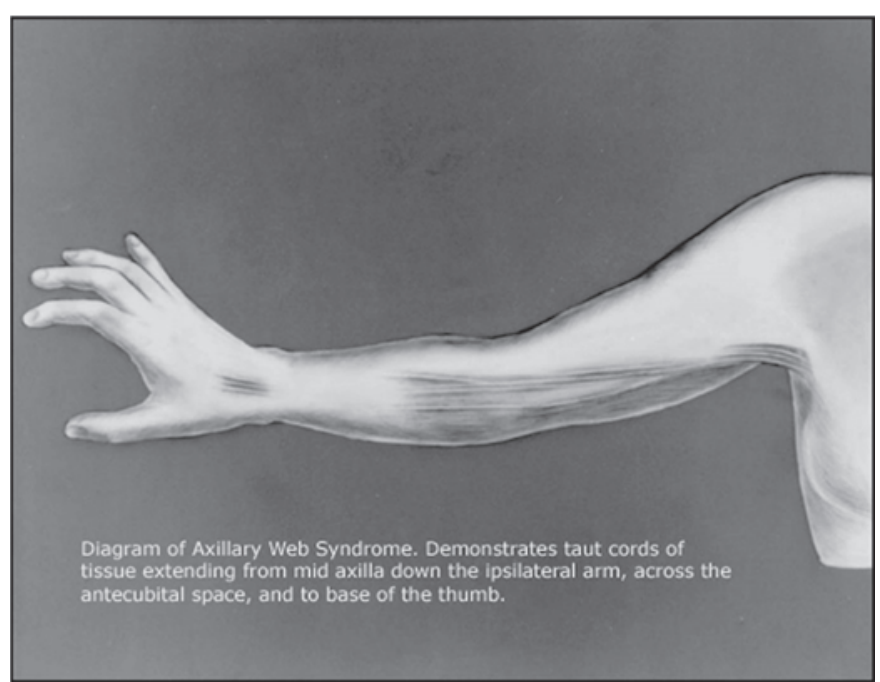

Fig. 1. Diagram of axillary web syndrome (free-to-use image downloaded from Google on August 18, 2017; originally published in [4]).

\section{Definition of Axillary Web Syndrome}

Initially defined as a syndrome 'characterized by axillary pain radiating down the ipsilateral arm, shoulder range-of-motion limitation, and an axillary web of tissue most obvious on physical examination when the patient tries to abduct her arm' [4], there remains the lack of a gold standard definition for AWS [12]. According to the authors of a recent systematic review of AWS, a web of axillary skin overlies 1 or more cords which have been described as feeling like ropes, bands, tendons, strings (or violin strings), or taut wires (fig. 1) [13]. These fibrous bands can extend down through the antecubital fossa and further into the volar surface of the forearm and wrist $[4,10]$. Typically, AWS occurs within the first 8 weeks after axillary surgery with symptoms resolving within the first 12 weeks postoperatively [12]. However, some investigators have reported appearance [6] or persistence [7] of cording well beyond that 12 -week window to as late as 2 years after axillary surgery [6].

Because shoulder abduction is often impaired by AWS $[4,13$, 14], the upper extremity range-of-motion necessary for undergoing chest and/or axillary radiation therapy may be compromised [12]. In a prospective cohort study of 85 breast cancer patients, Leidenius et al. [13] reported restricted shoulder motion in 16\% of those with AWS who had received sentinel lymph node biopsy (SLNB) only and in $64 \%$ of patients with AWS who underwent axillary clearance in addition to SLNB.

\section{Prevalence of Axillary Web Syndrome}

Across different studies, the prevalence of AWS varies widely. Although the initial study to define and describe AWS - a retrospective chart review - reported a frequency of only 6\% [4], a 2015 systematic review reported rates ranging from 10 to $85.4 \%$ across 6 prospective studies [12]. A prospective cohort study involving 964 breast cancer patients that was published subsequent to that review reported an AWS frequency of 35.9\% [11]. This rate is roughly comparable to the median frequency rate for the 17 prospective studies included in the earlier systematic review [12].

Several studies have compared the rates of AWS for women who have undergone SLNB to those of women who have had axillary lymph node dissection (ALND) $[6-9,13,15]$, positing that rates would be lower for the former group because of less disruption of the lymphatic system (as is true also for rates of lymphedema [16]). Table 1 shows that AWS occurs 1.7-7 times more frequently after ALND than after SLNB.

A recent meta-analysis showed that lymphedema was 3-4 times more frequent among women who had undergone axillary dissection than among those who had received SLNB [16]. Noteworthy also from this meta-analysis was the relatively low overall rate of lymphedema at $21.4 \%$ [16] as compared to AWS, with a median frequency of about $36-37 \%$ [12].

\section{Pathology Underlying the Axillary Web Syndrome}

In their report on the retrospective chart review in which AWS was first described, Moskovitz et al. [4] speculated that the syndrome resulted from disruption of the superficial lymphatic and blood vessels during axillary surgery. In their histological examination of the cords of 4 patients with AWS, they found dilated, thrombosed lymphatic vessels, or thrombosed superficial veins, or in some cases both.

In a 2015 systematic review of AWS [12], 5 level IV studies suggested that the underlying histology of AWS was likely due to thrombosed lymphatic vessels, with another study suggesting that the etiology of the cording was secondary to disruption of both veins and lymph vessels, as was initially proposed [4]. Although uncertainty persists regarding a definitive etiology, disruption of the lymphatic vessels was reported more commonly, although venous involvement cannot be ruled out [12].

\section{Risk Factors for Developing Axillary Web Syndrome}

Whereas being overweight or obese is a well-known risk factor for developing upper extremity lymphedema after breast cancer surgery [17], the reverse appears to be generally true for the development of AWS (i.e., thinner women are at greater risk). In a prospective cohort study involving 85 women with breast cancer, the median body mass index (BMI) for those with AWS was 23 as compared to a median BMI of 26 for those who did not develop the syndrome [13]. In another prospective study of 116 women, mean BMI for those with AWS was 25.1 versus 28.9 for those without AWS [7], whereas a third prospective study showed that overweight/obese women were $15 \%$ less likely to develop AWS than those with a normal BMI [8]. A third but smaller $(n=36)$ prospective cohort study showed a significant relationship between lower 
Table 1. Frequency of axillary web syndrome based on type of axillary surgery

\begin{tabular}{lll}
\hline $\begin{array}{l}\text { First author, year [ref.] } \\
\text { (participants, } n)\end{array}$ & $\begin{array}{l}\text { ALND } \\
\text { frequency, \% }\end{array}$ & $\begin{array}{l}\text { SLNB } \\
\text { frequency, \% }\end{array}$ \\
\hline Leidenius, 2003 [13] $(\mathrm{n}=85)$ & 72 & 10 \\
Bergmann, 2012 [8] $(\mathrm{n}=193)$ & 36 & 11.7 \\
O'Toole, 2013 [6] $(\mathrm{n}=308)$ & 54 & 27 \\
Wernicke, 2013 [15] $(\mathrm{n}=226)$ & 5.2 & 0.9 \\
Koehler, 2015 [9] $(\mathrm{n}=36)$ & 71 & 41 \\
\hline
\end{tabular}

ALND = Axillary lymph node dissection; SLNB = sentinel lymph node biopsy.

BMI and AWS in univariate analysis [6]. In contrast, a retrospective, descriptive design showed no significant association between AWS and BMI although the women with AWS had a lower mean BMI than those without (27.29 vs. 28.15); the authors commented that their failure to find a difference may have been due to the fact that the vast majority of women in their sample were overweight or obese [10].

Younger age has been shown also to be a risk factor for developing AWS (or cording) in 4 prospective cohort studies $[6-8,10]$. In that younger women with breast cancer typically have a lower BMI, it has been speculated that age and BMI are related in their risk contributions [7]. As noted in table 1, the number of lymph nodes removed and the extent of axillary surgery (i.e., ALND vs. SLNB) are the main risk factors for developing AWS. In their prospective cohort study of 308 patients with unilateral breast cancer, O'Toole et al. [6] showed in a multivariate analysis that both ALND $(p<0.0001)$ and younger age at diagnosis $(p=0.0005)$ were significant risk factors for the development of cording.

\section{Diagnosis of Axillary Web Syndrome}

The diagnosis of AWS is usually made clinically, with the cording and restricted range-of-motion often noticed first by the patient herself $[9,18,19]$. Visible or palpable rope-like cords are present in the axilla and the antecubital fossa, and sometimes extend down the forearm and into the wrist $[6,9]$. Other diagnostic criteria include accompanying pain and restricted upper extremity range-of-motion, especially in shoulder abduction and elbow extension [7]. If the cords are not readily visible, moving the shoulder into maximal abduction may elicit their appearance [7, 9]. A highly sensitive and specific self-assessment questionnaire was developed recently at the European Institute of Oncology as a screening tool for self-diagnosis by the patient [20].

In an attempt to characterize and diagnose AWS more definitively, Koehler et al. [18] undertook a prospective study of 36 patients, 17 of whom had been clinically diagnosed with AWS, by taking ultrasound images of the involved cords to compare to mirror images on the opposite uninvolved side. Although ultrasound was unable to identify a specific structure analogous to AWS, the authors concluded that this meant that the cords were not associated with venous thrombosis but more likely represented lym- phatic vessels that were tethered together and would thus not be identifiable by ultrasound [18], a finding corroborated by Leduc et al. [21] in a smaller series of patients based on a combination of clinical signs, ultrasound imaging, and magnetic resonance imaging.

\section{Management of Axillary Web Syndrome}

Recommended treatments for AWS are diverse, although often unsupported by research evidence. Management strategies range from simple reassurance that the condition will resolve on its own to preoperative or postoperative patient education about AWS and different types of massage and physical therapies (PT). Several individual case reports have described management of AWS to include: active and passive shoulder range-of-motion exercises incorporating gentle stretching $[19,22]$; mobilization of superficial fascia around the shoulder and trunk [19]; therapeutic massage combined with passive shoulder range-of-motion (dynamic angular petrissage) [23]; home shoulder stretching and range-of-motion exercise combined with $300 \mathrm{mg}$ of Aescuven ${ }^{\circledR}$ Forte (Cesra, BadenBaden, Germany) twice a day for 1 week [24]; and moist heat to the axilla and medial side of the upper arm [22].

Only recently has a prospective group intervention study appeared in the literature. In 2016, Cho et al. [25] published a randomized controlled trial comparing the effects of 4 weeks of PT combined with manual lymphatic drainage (MLD) to PT-only for 46 women with AWS. Unfortunately, there was no untreated control group. Both groups showed significant improvements in quality of life, shoulder flexion strength, arm function, and pain with significantly greater improvements in pain and arm volume reductions for the PT + MLD group [25]. The inclusion of MLD as an intervention and arm volume as an outcome measure is somewhat puzzling, as prospective cohort studies have shown that AWS is not a risk factor for development of lymphedema $[9,11]$.

Although a 2017 systematic review concluded that PT was effective in improving shoulder range-of-motion, resolving pain, and reducing the appearance of cording in patients with AWS [26], that review was based on only 4 studies, 2 of which were individual case reports. The authors concluded correctly, however, that additional randomized controlled trials are needed to examine the effects of PT [26].

\section{Future Research Directions}

Despite being described more than 15 years ago, there has been very little interventional research to determine if manual therapies for AWS are effective or if they are even necessary. To answer these questions, randomized trials comparing a no-treatment control group to $\mathrm{PT}$ and/or massage or to patient education/reassurance that the syndrome will resolve are needed.

Further studies are needed also to determine the exact etiology and pathophysiology of this understudied syndrome in an effort to 
elucidate what types of therapeutic or pharmacological treatments might be most beneficial as well as whether or not it could be prevented.

\section{Conclusion}

AWS remains a poorly understood condition that continues to mystify the patients that experience it as well as their health care providers [27]; AWS has been reported to be a more common sequela of SLNB/ALND than lymphedema, infection, or seroma [28]. Because this syndrome can develop after a patient's last surgical follow-up visit [22], it is important that family physicians, as well as oncologists and surgeons, be able to clinically diagnose the syndrome so that they can reassure their patients that the condition is usually self-limiting [29] and make referrals to a physical therapist if the patient's range-of-motion is especially compromised or if she is experiencing pain during everyday activities.

Based on the limited treatment research published to date, it appears that PT (stretching and strengthening exercises and soft tissue mobilization) might improve shoulder function and quality of life and decrease pain in patients with AWS [26]. Future randomized trials should include, however, untreated control groups to definitively support this suggestion.

Oncologists, breast surgeons, and family physicians need to make patients aware of this prevalent complication and inform them preoperatively about what to anticipate. Because primary care physicians have reported being confident in managing anxiety in survivors of breast cancer [30], their awareness of AWS is paramount as they are increasingly the main providers of follow-up care for these patients. However, in an excellent and very recent review article on follow-up treatment for breast cancer written as a practical guide for family physicians, no mention was made of AWS [3]; this is yet another example of how under-recognized this very common clinical complication actually is and how important it is for oncologists to educate both their patients and their medical colleagues about AWS.

\section{Disclosure Statement}

The author declares no competing interests.

\section{References}

1 World Health Organization: Breast cancer: prevention and control. Breast cancer burden. 2016. www.who. int/cancer/detection/breastcancer/en/index1.html (accessed 25 Oct 2016).

2 Grunfeld E, Levine MN, Julian JA, et al: Randomized trial of long-term follow-up for early-stage breast cancer: a comparison of family physicians versus specialist care. J Clin Oncol 2006;24:848-855.

3 Sisler J, Chaput G, Sussman J, Ozokwelu E: Follow-up after treatment for breast cancer: practical guide to survivorship care for family physicians. Can Fam Physician 2016;62:e578-e585.

4 Moskovitz AH, Anderson BO, Yeung RS, et al: Axillary web syndrome after axillary dissection. Am J Surg 2001;181:434-439.

5 Young AE: The surgical management of early breast cancer. Int J Clin Pract 2001;55:603-608.

6 O'Toole J, Miller CL, Specht MC, et al: Cording following treatment for breast cancer. Breast Can Res Treat 2013;140:105-111.

7 Torres Lacomba M, Mayoral Del Moral O, Coperias Zazo JL, et al: Axillary web syndrome after axillary dissection in breast cancer. Breast Cancer Res Treat 2009; 117:625-630.

8 Bergmann A, Mendes VV, de Almeida Dias R, et al: Incidence and risk factors for axillary web syndrome after breast cancer surgery. Breast Cancer Res Treat 2012;131:987-992

9 Koehler LA, Blaes AH, Haddad TC, et al: Movement function, pain, and postoperative edema in axillary web syndrome. Phys Ther 2015;95:156-163.

10 Fukushima KF, Carma LA, Borinelli AC, et al: Frequency and associated factors of axillary web syndrome in women who had undergone breast cancer surgery: a transversal and retrospective study. Springerplus 2015;4:112.
11 Wariss BR, Costa RM, Pereira AC, Koifman RJ, Bergmann A: Axillary web syndrome is not a risk factor for lymphoedema after 10 years of follow-up. Support Care Cancer 2017;25:465-470.

12 Yeung WM, McPhail SM, Kuys SS: A systematic review of axillary web syndrome. J Cancer Surviv 2015;9: 576-598.

13 Leidenius M, Leppänen E, Krogerus L, von Smitten K: Motion restriction and axillary web syndrome after sentinel node biopsy and axillary clearance in breast cancer. Am J Surg 2003;185:127-130.

14 Piper M, Guajardo I, Denkler K, Sbitany H: Axillary web syndrome: current understanding and new directions for treatment. Ann Plast Surg 2006;76(suppl 3):S227-231.

15 Wernicke AG, Shamis M, Sidhu KK, et al: Complication rates in patients with axillary negative nodes 10 years after local breast radiotherapy after either sentinel lymph node dissection or axillary clearance. Am J Clin Oncol 2013;36:12-19.

16 DiSipio T, Rye S, Newman B, Hayes S: Incidence of unilateral arm lymphoedema after breast cancer: a systematic review and meta-analysis. Lancet Oncol 2013; 14:500-515.

17 Ugur S, Arici C, Yaprak M, et al: Risk factors of breast cancer-related lymphedema. Lymphatic Res Biol 2013; 11:72-75.

18 Koehler LA, Hunter DW, Haddad TC, et al: Characterizing axillary web syndrome: ultrasonographic efficacy. Lymphology 2014;47:156-163.

19 Fourie WJ, Robb KA: Physiotherapy management of axillary web syndrome following breast cancer treatment: discussing the use of soft tissue techniques. Physiotherapy 2009;95:314-320.

20 Nevola Teixeira LF, Veronesi P, Lohsiriwat V, et al: Axillary web syndrome self-assessment questionnaire: initial development and validation. Breast 2014;23: 836-843.
21 Leduc $\mathrm{O}$, Fumière $\mathrm{E}$, Banse $\mathrm{S}$, et al: Identification and description of the axillary web syndrome (AWS) by clinical signs, MRI and US imaging. Lymphology 2014; 47:164-176

22 Tilley A, Thomas-Maclean R, Kwan W: Lymphatic cording or axillary web syndrome after breast cancer surgery. Can J Surg 2009;52:E105-E106.

23 Lewis PA, Cunningham JE: Dynamic angular petrissage as treatment for axillary web syndrome occurring after surgery for breast cancer: a case report. Int J Ther Massage Bodywork 2016;9:28-37.

24 Wei P, Zhu L, Chen K, et al: Axillary web syndrome following secondary breast-conserving surgery: a case report. World J Surg Oncol 2013;11:8.

25 Cho Y, Do J, Jung S, Kwon O, Jeon JY: Effects of a physical therapy program combined with manual lymphatic drainage on shoulder function, quality of life, lymphedema incidence, and pain in breast cancer patients with axillary web syndrome after axillary dissection. Support Care Cancer 2016;24:2047-2057.

26 Luz CMD, Deitos J, Siqueira TC, Palú M, Heck APF Management of axillary web syndrome after breast cancer: evidence-based practice. Rev Bras Ginecol Obstet 2017;39:632-639.

27 Bernas MJ: Axillary web syndrome, the lost cord, and lingering questions. Lymphology 2014;47:153-155.

28 Nieves Maldonado SM, Pubul Núñez V, Argibay Vázquez S, Macías Cortiñas M, Ruibal Morell Á: Axillary web syndrome following sentinel lymph node biopsy for breast cancer. Rev Esp Med Nucl Imagen Mol 2016;35:325-328.

29 Aydogan F, Belli AK, Baghaki S, et al: Axillary web syndrome after sentinel node biopsy. Breast Care (Basel) 2008;3:277-278.

30 Smith SL, Wai ES, Alexander C, Singh-Carlson S: Caring for survivors of breast cancer: perspective of the primary care physician. Curr Oncol 2011;18:e218-226. 